\title{
Leukocytes and Interferon in the Host Response to Viral Infections
}

\author{
II. Enhanced Interferon Response of Leukocytes from Immune Animals \\ LOWELL A. GLASGOW \\ Departments of Microbiology and Pediatrics, University of Rochester School of Medicine and Dentistry, \\ Rochester, New York
}

Received for publication 5 February 1966

\begin{abstract}
Glasgow, Lowell A. (University of Rochester School of Medicine and Dentistry, Rochester, N.Y.). Leukocytes and interferon in the host response to viral infections. II. Enhanced interferon response of leukocytes from immune animals. J. Bacteriol. 91 :2185-2191. 1966. - The production of interferon was studied under in vitro conditions in peritoneal leukocytes or macrophages from mice immunized with Chikungunya virus (CV). Cultures of leukocytes obtained from animals immune to $\mathrm{CV}$ produced 2- to 10-fold greater amounts of interferon when exposed to an inoculum of CV than similar cell preparations from nonimmune, control animals. The viral inhibitor produced in increased quantity by $\mathrm{CV}$-immune leukocytes had the biological and biochemical properties of interferon. The enhanced interferon production was inhibited by actinomycin $\mathrm{D}$. This response of immune leukocytes was specific, and was initiated only by CV; it was not observed in leukocytes from animals immunized against other viruses which were challenged with $\mathrm{CV}$. The presence of neutralizing antibody could not be related to this response. The observed increase in interferon production was not dependent upon an enhanced virus uptake. The data are presented as a possible new dimension of the "immune response" and may suggest a mechanism for the phenomenon of "tissue immunity."
\end{abstract}

Evidence has been presented by a number of investigators which suggests that interferon may be a significant factor in host resistance to virus infections. These data were reviewed extensively in 1963 by Baron (1) and, more recently, by Glasgow (6). The majority of these studies, however, have been concerned with the interferon response of the host during primary infection, and few data have been reported regarding the induction of interferon production by virus on re-exposure of the immune host. The purpose of the present investigation, therefore, was to study interferon production by the immune animal on reinfection by a virus. During the course of these studies, it was noted that peritoneal leukocytes or macrophages obtained from animals immunized against Chikungunya virus $(\mathrm{CV})$ produced a significantly greater quantity of interferon than nonimmune leukocytes when challenged in vitro with the same virus. The data presented in this report deal primarily with this altered response of peritoneal leukocytes from immune animals.

\section{Materials AND Methods}

Virus. Vesicular stomatitis virus (VSV), Indiana strain, was obtained from the American Type Culture Collection. Stock virus pools used for interferon assays were grown in $\mathrm{L}$ cells, and titered approximately $2 \times 10^{6}$ plaque-forming units (PFU) per $\mathrm{ml}$ by use of plaque assay in $\mathrm{L}$ cells.

A standard reference strain of CV was obtained from Philip Russell, Walter Reed Army Medical Center. Stock virus was prepared from the brains of infected suckling mice, made into a $10 \%$ suspension, and assayed by the plaque method in primary rat embryo fibroblasts. Virus pools used for immunization of animals or induction of interferon production titered approximately $10^{7} \mathrm{PFU} / \mathrm{ml}$.

A large plaque mutant (EMCr) of encephalomyocarditis virus (EMC), originally obtained from $\mathrm{K}$. K. Takemoto at the National Institutes of Health, was prepared and assayed in $\mathbf{L}$ cells.

Mice. All animals used in these experiments were ex-breeder, female, random-bred, CD-1 mice obtained from the Charles River Mouse Farms.

Cells. L cells (clone 929) were from an established line of mouse fibroblast cells obtained from Piero 
Balduzzi, University of Rochester. Leukocyte preparations were obtained as previously described (7).

Media. All tissue cells were maintained in Eagle's minimal essential medium (MEM) with 5 to $10 \%$ calf serum. The medium used for plaque assays was Eagle's MEM with $0.9 \%$ Noble agar (Difco) and 5\% calf serum.

Virus titration. CV was assayed in primary or secondary rate embryo fibroblasts (REF) by use of a plaque method. Samples of virus to be assayed were serially diluted, and $0.2 \mathrm{ml}$ was inoculated onto monolayer cultures in 60-mm plastic petri dishes (Falcon Plastic, Div. of B-D Laboratories, Los Angeles, Calif.). The inoculum was allowed to adsorb for 1 $\mathrm{hr}$, and the preparation was overlaid with $5 \mathrm{ml}$ of agar medium. A second overlay, containing neutral red to make a final concentration of 1:20,000, was made on the 2 nd or $3 \mathrm{rd}$ day, and plaques were counted.

Interferon assay. Samples were prepared and assayed for interferon activity as previously described (7). Interferon titers are expressed as the reciprocal of the dilution which inhibits $50 \%$ of a challenge inoculum of approximately 50 PFU of VSV.

Antibody assay. Anti-CV antibody levels in immunized mice were determined by use of a plaqueneutralization method. Mouse serum was obtained by orbital bleeding, and serial fourfold dilutions were made in Eagle's MEM. Samples were incubated at room temperature for $0.5 \mathrm{hr}$ with approximately 100 PFU of CV, and were inoculated onto $60-\mathrm{mm}$ plastic petri dishes containing a monolayer of primary or secondary REF. The remainder of the assay was carried out as described for the CV assay, and the serum dilution neutralizing $50 \%$ of the inoculum was determined.

\section{RESULTS}

Interferon production by leukocytes from immune animals. CD-1 mice were immunized against $\mathrm{CV}$ by three to six intraperitoneal injections of $10^{6}$ to $5 \times 10^{6} \mathrm{PFU}$ of virus. At least 1 month after the last injection, peritoneal leukocytes were harvested from two to four $\mathrm{CV}$ immune mice, and a similar number of matched control animals, as described previously (7). The harvested cells were sedimented at 1,200 $\mathrm{rev} / \mathrm{min}$, resuspended in Eagle's MEM, and counted, and $3 \times 10^{6}$ to $5 \times 10^{6}$ cells were distributed in plastic petri dishes in a total volume of $6 \mathrm{ml}$ of Eagle's MEM with $10 \%$ calf serum. The leukocyte inoculum was adjusted, as indicated by the cell count, so that cultures in each group contained an equal number of cells. Leukocyte cultures were infected with $2 \times 10^{6}$ to $5 \times 10^{6}$ PFU of CV, and supernatant fluids were harvested for interferon assay at varying intervals from 2 to $48 \mathrm{hr}$ after infection.

The immune status of mice immunized with CV was confirmed by determining the level of neutralizing antibody in the serum of experi- mental animals. After three to six injections of virus, all animals tested had levels of anti-CV antibody ranging from $1: 640$ to $1: 5,120$.

Data from 1 of the 27 experiments on which this report is based are summarized in Fig. 1. Cultures of immune leukocytes produced greater quantities of interferon than nonimmune cells, as determined by assay of antiviral activity at all time intervals from 4 to $24 \mathrm{hr}$ after CV challenge. The final yield of interferon varied greatly among experiments, but in most instances the immune leukocytes produced two to ten times as much interferon as a similar number of nonimmune cells. The range of final yields from a number of experiments is illustrated in Table 1 . These differences resulted from the fact that the experiments were carried out over an 18-month period, during which time different $\mathrm{CV}$ pools with varying titers were utilized, and the number of leukocytes in different experiments ranged from $3 \times 10^{6}$ to $6 \times 10^{6}$ per plate.

These data indicate that peritoneal leukocytes or macrophages from an immunized animal have the capacity for an enhanced response, in terms of interferon production, on secondary exposure to $\mathrm{CV}$. Further studies were designed to delineate the nature of this response.

Characteristics of interferon produced by immune leukocytes. The biochemical and biological properties of the interferon produced by the leukocytes from immune animals were determined and compared with those of nonimmune leukocytes and nonphagocytic mouse cells (a continuous line of mouse embryo fibroblasts).

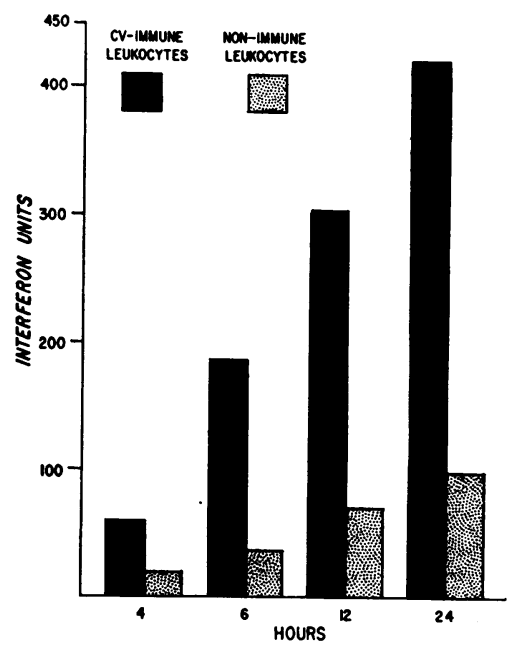

FIG. 1. Comparison of interferon production after exposure to $C V$ in $C V$-immune and nonimmune mouse leukocytes. An enhanced interferon response is demonstrated in cells from immune animals. Interferon titers are expressed as units per milliliter. 
TABLE 1. Interferon production in $C V$-immune and nonimmune leukocytes exposed to $C V$

\begin{tabular}{r|c|c}
\hline & \multicolumn{2}{|c}{ Interferon production } \\
\cline { 2 - 3 } Expt no. & CV-immune & Nonimmune \\
\cline { 2 - 3 } V & units & units \\
VI & $>100$ & 15 \\
XII & 80 & 8 \\
XIII & 420 & 98 \\
XIV & 1,000 & 160 \\
XVIII & 650 & 185 \\
XXII & 45 & 20 \\
XXXIII & 223 & 105 \\
& 89 & 27 \\
\hline
\end{tabular}

The antiviral inhibitor from all three sources had the following properties: (i) stable at $p \mathrm{H} 2$ for 24 $\mathrm{hr}$; (ii) loss of antiviral activity in presence of trypsin; (iii) partial loss of activity when heated at $56 \mathrm{C}$ for $30 \mathrm{~min}$; (iv) complete loss of activity when heated at $70 \mathrm{C}$ for $30 \mathrm{~min}$; (v) failure to sediment at $90,000 \times g$ for $1 \mathrm{hr}$; (vi) antiviral activity against a diverse group of viruses; (vii) species specificity, as determined by lack of activity in chick embryo tissue culture; (viii) no loss of activity when a culture which was treated with the inhibitor was washed prior to virus challenge; and (ix) no direct antiviral activity when inhibitor and challenge virus were mixed and incubated together for $1 \mathrm{hr}$ at $37 \mathrm{C}$. These data indicate that the properties of the inhibitor produced in greater quantity by the leukocytes from immune animals conform with those of interferon.

Inhibition by actinomycin $D$. The production of interferon is inhibited by actinomycin $\mathrm{D}(8,9$, $11,16,17)$. To characterize further the inhibitor produced by leukocytes from immune animals, cultures of peritoneal leukocytes from immune and nonimmune animals were treated with 2.0 and $0.2 \mu \mathrm{g} / \mathrm{ml}$ of actinomycin $\mathrm{D}$ and then challenged with CV. Supernatant fluids were harvested at $24 \mathrm{hr}$, treated with acid, and assayed for interferon activity with EMC virus as the challenge agent. The data presented in Table 2 show that the enhanced interferon production by immune leukocytes is inhibited by actinomycin $D$, and further confirm the evidence that the interferon-like substance produced by control and immune leukocytes is, in fact, interferon.

Specificity of enhanced interferon response. To determine whether the enhanced interferon production was a specific response to the immunizing agent, peritoneal leukocytes were harvested from CV-immune and control, nonimmune animals, and replicate cultures of cells from both groups
TABLE 2. Effect of actinomycin $D$ on interferon production in $C V$-immune and nonimmune leukocytes

\begin{tabular}{cc}
\hline Group & $\begin{array}{c}\text { Interferon } \\
\text { produc- } \\
\text { tion }\end{array}$ \\
\hline units
\end{tabular}

Immune leukocytes............ 240

Immune leukocytes $+0.2 \mu \mathrm{g} / \mathrm{ml}$ of actinomycin D............... 20

Nonimmune leukocytes ............ $\quad 110$

Nonimmune leukocytes $+0.2 \mu \mathrm{g} / \mathrm{ml}$ of actinomycin D.............. 18

were challenged with $\mathrm{CV}$, Sindbis, or vaccinia virus. The results of assays of interferon activity in supernatant fluids (Table 3) demonstrate that the enhanced interferon response occurred only in the culture challenged with $\mathrm{CV}$. As part of another study, a similar enhancement of interferon production by leukocytes from mice immunized against vaccinia virus has been demonstrated (Glasgow, in preparation). Thus, the specificity of increase of interferon production by $\mathrm{CV}$-immune cells was further defined by showing that similar final yields of interferon were obtained in cultures of leukocytes from vacciniaimmune and control animals challenged with CV.

These data indicate that the increased yield of interferon produced in leukocytes from immune animals is a specific response, and suggest either an altered virus-cell interaction resulting in enhanced virus uptake by the immune cell, or the possibility that leukocytes or macrophages are capable of responding to a secondary exposure of a virus with an enhanced interferon production in a fashion analogous with that of the anamnestic antibody response to a foreign antigen.

Virus adsorption by $C V$-immune and control leukocytes. Replicate cultures of leukocytes from CV-immune and nonimmune control animals were set up as described and infected with $2 \times 10^{6}$ PFU of CV. Samples of culture medium were

TABLE 3. Specificity of enhanced interferon response in CV-immune and nonimmune leukocytes

\begin{tabular}{c|l|c}
\hline \multicolumn{1}{c|}{ Group } & Challenge virus & $\begin{array}{l}\text { Interferon } \\
\text { production }\end{array}$ \\
\cline { 3 - 3 } CV-immune leukocytes & CV & units \\
& Vaccinia & 250 \\
& Sindbis & 4 \\
Nonimmune leukocytes & CV & 55 \\
& Vaccinia & 5 \\
& Sindbis & 6 \\
\hline
\end{tabular}




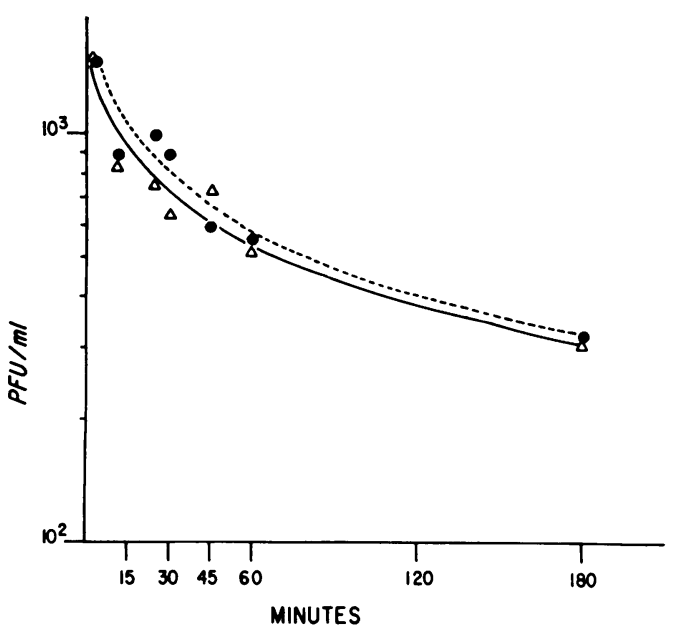

FIG. 2. Demonstration of a similar rate of adsorption or phagocytosis of $\mathrm{CV}$ in cultures of leukocytes from $C V$-immune $(\bigcirc)$ and control nonimmune $(\triangle)$ animals. Nonadsorbed $\mathrm{CV}$ in supernatant culture fluids is expressed as plaque-forming units per milliliter. collected and centrifuged, and supernatant fluids were assayed for unadsorbed virus. The immune status of the animals used in this experiment was confirmed by determination of neutralizingantibody titer of pooled sera collected at the time peritoneal leukocytes were harvested. Cage mates of the animals used in this experiment were shown in simultaneous experiments to manifest the enhanced interferon response (experiment XIV in Table 1). As shown in Fig. 2, CV was adsorbed by leukocytes from immune and control animals at identical rates. It would appear, therefore, that enhanced adsorption by immune cells is not responsible for the observed enhancement of interferon production.

The concomitant development of immunity, as characterized by high levels of neutralizing antibody, and of the capacity for an enhanced interferon response suggested a possible relationship between the presence of antibody capable of complexing with $\mathrm{CV}$ and the observed increase in interferon production. This possibility was considered in the following experiments

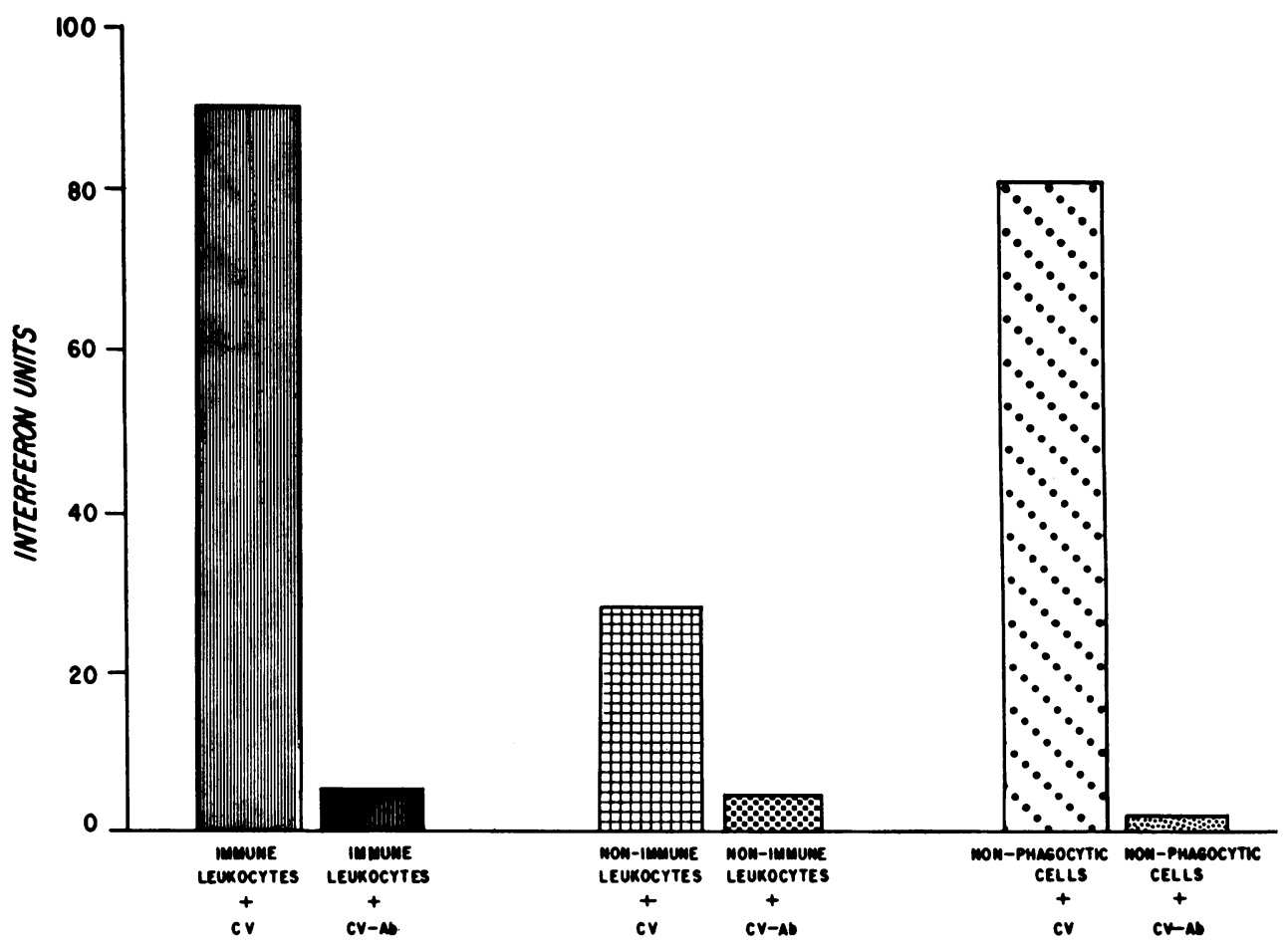

FIG. 3. Inhibition of interferon production by $C V$-neutralizing antibody in cultures of both $C V$-immune and nonimmune leukocytes, as well as in a continuous line of mouse fibroblasts. Final yields of interferon are compared in supernatant fluids harvested at $24 \mathrm{hr}$ from cultures exposed to Chikungunya virus-normal mouse serum (CV) or Chikungunya virus-anti Chikungunya antisera $(C V-A b)$. 
carried out in vitro with hyperimmune anti-CV mouse serum

Replicate cultures containing equal numbers of peritoneal leukocytes from either immune or nonimmune mice were established. A third group of plates containing monolayers of a continuous line of mouse embryo fibroblasts (3-B) was also set up. Two samples of stock CV were incubated at room temperature for $15 \mathrm{~min}$ with normal mouse serum or anti-CV immune serum (plaque inhibiting titer, 1:5,120), and samples of each preparation were used to challenge the three groups of cultures. After $24 \mathrm{hr}$, supernatant fluids were harvested and assayed for interferon activity. The data presented in Fig. 3 indicate a high yield of interferon from the continuous line of mouse fibroblasts. Since the primary purpose of the experiment was to compare interferon production in (i) immune versus nonimmune leukocytes, and (ii) virus versus virus-antibody complexes, the number of cells in the continuous line cultures was not adjusted to equal the leukocyte cell counts. No conclusions, therefore, may be drawn from these data concerning interferon production in leukocytes in comparison with nonphagocytic cells.

The complexing of virus and antibody resulted in a striking reduction in the stimulation of interferon production in all cell groups. These data indicated that virus complexed with antibody has a significantly impaired capacity to function as a stimulus of interferon production, and strongly suggest that the presence of anti-CV antibody is not causally related to the enhanced interferon response in immune cells.

Although the data (Fig. 2) indicated that an increased rate of adsorption was not correlated with the enhanced interferon response of $\mathrm{CV}$ immune cells, the possibility still existed that cell-bound anti-CV antibody could alter virushost cell interaction after adsorption. To test this hypothesis, normal, nonimmune peritoneal leukocytes were exposed to normal and anti-CV immune serum. Cells were then sedimented, washed, resuspended in culture medium, and challenged with CV. The results of this experiment are presented in Fig. 4. The exposure of normal, nonimmune cells to anti-CV antibody resulted in a slightly diminished capacity to respond to $\mathrm{CV}$ with the production of interferon. From the data previously presented, which showed that virus-antibody complexes induced only negligible interferon production, it would be expected that adsorption of some anti-CV antibody on the surface of normal nonimmune leukocytes might result in the observed decreased stimulation of interferon production.

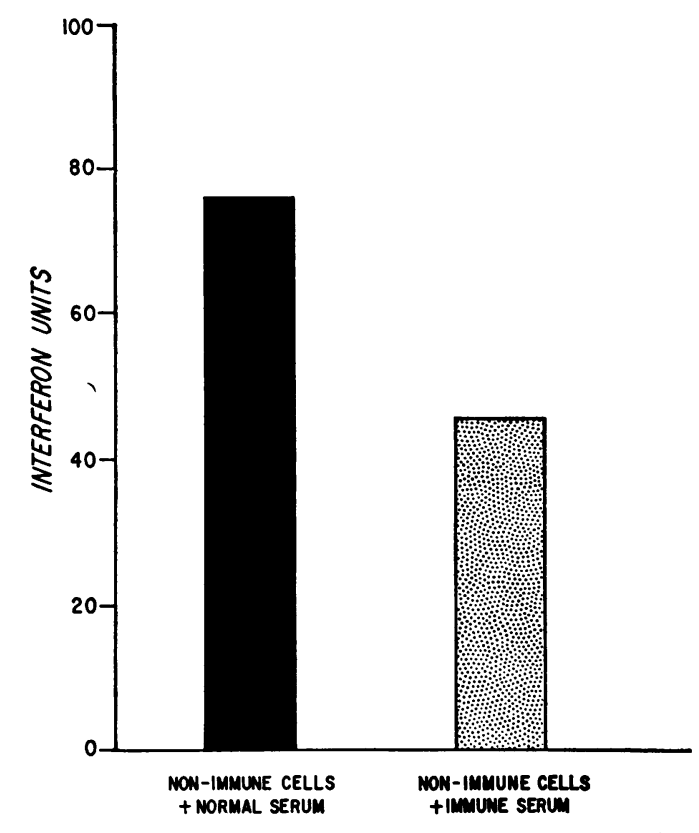

FIG. 4. Effect of incubating nonimmune mouse leukocytes with anti-CV immune sera prior to challenge with $\mathrm{CV}$. The adsorption of neutralizing antibody on nonimmune leukocytes resulted in a moderate decrease in final interferon yields.

\section{Discussion}

The data presented from a large series of experiments demonstrate that leukocytes or macrophages from CV-immune animals manifest an altered response in vitro on re-exposure to the same virus, and suggest that leukocytes may have the capacity to respond to such a secondary exposure with an enhanced production of interferon. The concept of "tissue or cellular immunity," i.e., the enhanced resistance of the immune host apart from demonstrable neutralizing antibody, has been considered and discussed for many years, but concrete mechanisms related to this type of resistance have not been demonstrated. Douglas and Smith (3) interpreted their studies of vaccinal immunity in rabbits to indicate that immune tissue differs from normal tissue. These workers found that the leukocytes or cells from spleen and testes of vaccinia-immune animals had an enhanced capacity to prevent initiation of vaccinia virus lesions, when compared with nonimmune cell preparations. From this evidence, they concluded that "there is a cellular immunity against vaccinia virus as well as a humoral immunity." These conclusions were supported by Fairbrother (4), who inoculated different combinations of vaccinia virusserum-leukocyte mixtures intracerebrally into 
rabbits. These data, however, are difficult to interpret, since small numbers of animals were used and the most significant degree of protection was observed with combinations of immune serum and leukocytes.

Ginder (5) has reported a series of experiments in which mixtures of fibroma virus and fibromaimmune serum with leukocytes or macrophages were inoculated into rabbits. He found that the addition of fibroma-immune cells enhanced the neutralizing capacity of antifibroma serum. This increase in neutralization was a function of living cells and could not be explained by the presence of cell-bound antibodies, but the mechanism by which fibroma-immune macrophages exerted this effect was not elucidated. More recently, Steinberger and Rights (15) demonstrated that cultures of spleen cells from vaccinia-immune rabbits were less susceptible to infection by vaccinia virus than nonimmune control cultures. Furthermore, virus growth was limited in the immune spleen cultures, but not in similar cultures of immune kidney cells. With the use of plaque-neutralization techniques, antibody could not be demonstrated in the culture from the more resistant spleens. The occurrence of this resistance in cultures of spleen cells, but not of kidney cells, suggests that the observed phenomenon may have been dependent upon the presence of reticuloendothelial-system cells in the spleen. This decreased susceptibility of immune spleen cells in the absence of detectable antibody strongly suggests an altered virus-host interaction at the cellular level, and may be explained in terms of the results presented with immune macrophages.

Kempe (10) has reported a case of progressive vaccinia in a 1-year-old child, which failed to respond to large doses of hyperimmune $\gamma$-globulin. The continued spread of the infection was finally arrested by the injection of leukocytes and a lymph-node cell suspension from immune donors, both intravenously and at the leading edge of the lesion. It was proposed that the delayed hypersensitive reaction, mediated by the transferred immune cells, might contribute to the process of limiting the further spread of the virus and to its eventual elimination. In view of the present results, however, it would not seem unreasonable to suggest that enhanced production of interferon by the transferred leukocytes may have been a factor in the outcome of the infection. Preliminary studies in this laboratory with vaccinia virus suggest that a similar enhanced interferon response is, in fact, seen in cells from vaccinia-immune animals (Glasgow, in preparation).
Other workers, most notably Sabin (14) and Beard and Rous (2), considered the same problem as Douglas and Smith (3), i.e., the role of leukocytes in vaccinia virus infections of rabbits, and failed to demonstrate an enhanced antivaccinal or "virucidal" activity in leukocytes from immune animals. The reasons for these discrepancies are not readily apparent, although virus-host systems, techniques, and methods of assay have varied widely among the different investigators, and conditions have certainly not been optimal in many of these experiments for the demonstration of interferon activity.

Evidence has been presented that the observed enhancement of interferon production was not related to an increased rate of virus adsorption. In a different experimental system, Roberts (13) has reported an apparent increase in susceptibility of immune macrophages to ectromelia virus, which he attributed to a nonspecific enhancement of phagocytic activity. This effect was noted in cultures of peritoneal macrophages harvested from animals immunized by the intraperitoneal route, and wore off by 21 days after inoculation. In contrast, the present studies were carried out in animals immunized by the subcutaneous route, and peritoneal exudates were usually harvested between 3 and 8 weeks after the last virus booster. It would appear unlikely, therefore, that the phenomenon observed by Roberts was a factor in the results obtained in this investigation.

The significance of the data reported, in terms of host resistance of immune animals, remains an open question. Although interferon may be implicated in the process of virus elimination during primary infection, there is no evidence that it contributes to host resistance during reinfection. At our present stage of understanding, extrapolation from the experimental model to the situation in the immune host is obviously hazardous. However, the results presented in this study provide a possible mechanism for an enhanced tissue response on reinfection. The failure of neutralized virus to induce the production of interferon in vitro confirms the in vivo work of Youngner and Stinebring (18) and Pollikoff (12), and suggests a limited in vivo function of the observed phenomenon. Although an enhanced interferon response by immune leukocytes may not be a major factor in resistance, it may possibly play a role at local sites of infection or in situations where cell to cell transmission may permit persistence of the virus in the face of neutralizing antibody. Studies are in progress to define further the nature of this apparent capacity for "recognition" in the processes involved in the regulation of interferon produc- 
tion, to determine whether the response is limited to the $\mathrm{CV}$ model or is a general phenomenon, and to investigate its possible in vivo significance.

\section{ACKNOWLEDGMENTS}

This investigation was supported by Public Health Service grant 06388-01 from The National Institute of Allergy and Infectious Diseases.

The author wishes to express his appreciation to Yetta Beach and Nancy Gurowitz for their able technical assistance, and to Marilyn Graver for the preparation of the manuscript.

\section{Literature Cited}

1. BARON, S. 1963. Mechanism of recovery from viral infection. Advan. Virus Res. 10:39-64.

2. Beard, J. W., AND P. Rous. 1938. The fate of vaccinia virus on cultivation in vitro with Kupffer cells (reticuloendothelial cells). J. Exptl. Med. 67:883-910.

3. Douglas, S. R., AND W. SMIth. 1930. Vaccinal immunity in rabbits by means of in vitro methods. Brit. J. Exptl. Pathol. 11:96-111.

4. Fairbrother, R. W. 1933. The production of immunity to vaccinia virus by mixtures of immune serum and virus and the importance of phagocytosis in antivaccinal immunity. $\mathbf{J}$. Pathol. Bacteriol. 36:55-64.

5. GINDER, D. R. 1955. Resistance to fibroma virus infection. The role of immune leukocytes and immune macrophages. J. Exptl. Med. 101: $43-58$.

6. Glasgow, L. A. 1965. Interferon: a review. J. Pediat. 67:104-121.

7. Glasgow, L. A. 1965. Leukocytes and interferon in the host response to viral infections. I. Mouse leukocytes and leukocyte-produced interferon in vaccinia virus infection in vitro. J. Exptl. Med. 121:1001-1018.
8. Heller, E. 1963. Enhancement of Chikungunya virus replication and inhibition of interferon production by actinomycin D. Virology 21: 652-656.

9. Ho, M. 1964. Identification and "induction" of interferon. Bacteriol. Rev. 28:367-381.

10. KemPe, C. H. 1960. Studies on smallpox and complications of smallpox vaccination. Pediatrics 26:175-189.

11. Levy, H. B., D. Axelrod, and S. Baron. 1965. Messenger RNA for interferon production. Proc. Soc. Exptl. Biol. Med. 118:384-385.

12. Pollikoff, R., M. Lieberman, AND N. E. Lem, 1965. Role of interferon and partial immunity in influenza virus infection of mouse lung. Proc. Soc. Exptl. Biol. Med. 119:790-793.

13. Roberts, J. A. 1964. Growth of virulent and attenuated ectromelia virus in cultures macrophages from normal and immune mice. $J$. Immunol. 92:837-842.

14. SABIN, A. B. 1935. The mechanism of immunity to filterable viruses. III. The role of leukocytes in immunity to vaccinia. Brit. J. Exptl. Pathol. 16:158-168.

15. Steinberger, A., AND F. L. Rights. 1963. Effect of immunization on tissue susceptibility to vaccinia virus in vitro. Virology $21: 402-410$.

16. WAGNER, R. R. 1963. Interferon control of viral infection. Trans. Assoc. Am. Physicians 76: 92-101.

17. WAGNER, R. R. 1964. Inhibition of interferon biosynthesis by actinomycin D. Nature 204: 49-51.

18. Youngner, J. S., AND W. R. Stinebring. 1965. Interferon appearance stimulated by endotoxin, bacteria, or viruses in mice pretreated with Escherichia coli endotoxin or infected with Mycobacterium tuberculosis. Nature 208:456458. 